\title{
Pituitary injury and persistent hypofunction resulting from a peripartum non-hemorrhagic, vaso-occlusive event
}

\author{
Anita Kuriya', David V Morris² and Michael H Dahan' \\ Division of Reproductive Endocrinology and Infertility, Departments of ${ }^{1}$ Obstetrics and Gynecology \\ ${ }^{2}$ Division of Endocrinology, McGill University Health Center, 687 Pine Avenue West F 6.58, Montreal, \\ Quebec, Canada H3A 1A1
}

Correspondence should be addressed to M H Dahan

Email

dahanhaim@hotmail.com

\section{Summary}

Cerebral vascular accidents are caused by vasospasm when induced by preeclampsia or by dopamine agonists. However, six arteries nourish the pituitary and prevent against vasospasm-induced damage, which up until now has not been thought to occur. Bromocriptine was used to arrest lactation in a 31-year-old with secondary amenorrhea following preeclampsia and fetal demise at 28 weeks gestation. Tests and history revealed panhypopituitarism not associated with hemorrhage or mass infarction but instead caused by vasospasm. The present study is the first report of pituitary damage from a nonhemorrhagic, vaso-occlusive event in the literature. In keeping with Sheehan's and Simon's syndromes, we have named pituitary damage resulting from vaso-occlusion as Dahan's syndrome, and a literature review suggests that it may be a common and previously overlooked disorder.

\section{Learning points:}

- Vasospasm can cause damage to the pituitary gland, although it was not previously believed to do so.

- Preeclampsia and the use of a dopamine agonist, particularly in the peripartum state, may trigger vasospasm.

- Vasospasm resulting from dopamine agonists may be a common cause of injury to the pituitary gland, and it may have been overlooked in the past.

\section{Background}

During pregnancy, the pituitary gland undergoes hypertrophy and enlarges to compensate for increased hormone production during the intra-partum and postpartum periods. The circle of Willis is a physiologic compensatory mechanism to protect the pituitary gland from blood loss, which is a common phenomenon at delivery. Inflow to the circle occurs from the right and left anterior, middle, and posterior cerebral arteries. The fact that six arteries supply blood to the pituitary protects this gland from insufficiency when an artery or, in theory, even two arteries are occluded because of stroke, vasospasm, or significant postpartum hemorrhage. Having so many arteries feed the gland ensures that some blood flow continues during peripartum severe shock.

Preeclampsia has been shown to result in cerebral vascular thrombosis during pregnancy (1) (2). One study showed that risk factors for postpartum cerebral vascular disease, including ischemic stroke, intracranial hemorrhage, cerebral venous sinus thrombosis, pituitary apoplexy, carotid/vertebral artery dissection, hypertensive encephalopathy, or other acute cardiovascular disease that occurred after hospital discharge but before 6 weeks after delivery, were eclampsia (odds ratio (OR) 10.1; 95\% CI $3.09,32.8$ ) and preeclampsia (OR 2.1; 95\% CI 1.6, 2.8), 
among other factors, including coagulation disorders and African origins (3). However, that study did not add to our understanding of the mechanisms that cause these disorders. The mechanism that underlies preeclampsiacaused vascular thrombosis is thought to be vasospasm, which also puts the patients at risk for eclamptic seizures (4). Dopamine agonists have also been noted to place patients at risk for postpartum vaso-occlusive episodes (2). The case in the present study is the first to report pituitary damage from a non-hemorrhagic vasospasmic event; it is thus a combination of severe preeclampsia and a postpartum dopamine agonist.

\section{Case presentation}

A 31-year-old African American G2P2L1 type 2 diabetic (DM2) presented with a 3-year history of secondary infertility and amenorrhea without any headaches. She had been diagnosed with DM2 6 years earlier after being tested for it because of her strong family history of DM2. Her condition was managed with short-acting insulin lispro: 14 units before breakfast, 14 units before lunch, and 14 units before dinner as s.c. injections. She also received an injection of 12 units of neutral protamine Hagedorn long-acting insulin at bedtime. Sliding scale lispro insulin was also used as needed. She demonstrated no evidence of organ injury.

The patient did not report any difficulty in conceiving her first two pregnancies. The amenorrhea presented in the immediate postpartum period, and she failed to have any menses after delivery. She had undergone a successful induction of labor at 28 weeks gestation for severe preeclampsia and fetal demise at another institution. There was no history of eclampsia, HELLP (hemolysis, elevated liver enzymes, and low platelets) syndrome, postpartum hemorrhage, infection, fever, or curettage. Her liver enzymes and platelet count were normal throughout her admission. However, she was given bromocriptine (5 mg daily for 30 days) postpartum to prevent lactation because of the fetal demise. Two days after delivery, she complained of severe headache and transient left-sided weakness. A computed tomography (CT) scan and CT arteriogram of the brain were ordered. The CT scan did not find any masses, lesions, vascular accidents, hemorrhages, or sinus thrombosis. The pituitary gland was normal size, and no adenomas or deviations were noted. The arteriogram did not detect any vascular occlusions. However, vascular flow was noted to be diffusely decreased, particularly at the distal ends of arteries, which is consistent with diffuse spasm. In spite of this finding, the bromocriptine was continued. No delivery of vasodilators was recorded.
The patient described her amenorrhea as dating from that period onward. She denied any history of bitemporal hemianopsia, diploplia, or any other vision problems during or after the pregnancy. Loss of consciousness, frequent urination, and polydipsia were also denied.

\section{Investigation}

Upon physical examination, the patient had an elevated BMI $\left(36 \mathrm{~kg} / \mathrm{m}^{2}\right)$. Her HbA1c level was $7.9 \%$. Her initial ultrasound showed an anatomically normal uterus with an antral follicle count of 11 . Random hormonal test results showed a serum with $3.0 \mathrm{IU} / 1$ follicle-stimulating hormone (FSH; follicular phase: 2-15 IU/l, mid-cycle: 5-20 IU/l, luteal phase: 2-10 IU/l, and menopause: 25-135 IU/l), $3.0 \mathrm{IU} / \mathrm{l}$ luteinizing hormone (LH; follicular phase: $2-10 \mathrm{IU} / \mathrm{l}$, midcycle: 15-100 IU/l, luteal phase: 1-10 IU/l, and menopause: 10-60 IU/l), 41 pmol/1 estradiol ( $\mathrm{E}_{2}$; normal: non-pregnant $50-1800 \mathrm{pmol} / \mathrm{l}$ and menopausal $<18-200 \mathrm{pmol} / \mathrm{l})$, $1.52 \mathrm{mIU} / \mathrm{l}$ thyrotrophin (TSH; normal: $0.40-4.40 \mathrm{mIU} / \mathrm{l}$ ), $17 \mathrm{pmol} / \mathrm{l}$ free thyroxine (normal: $10-23 \mathrm{pmol} / \mathrm{l}$ ), $0.22 \mathrm{nmol} / \mathrm{l}$ total testosterone (normal: $0.35-2.70 \mathrm{nmol} / \mathrm{l}$ ), and $3.0 \mu \mathrm{g} / \mathrm{l}$ prolactin (normal: 3.3-26.7 $\mu \mathrm{g} / \mathrm{l}$ ). Repeat random analysis showed a serum with $3.7 \mathrm{IU} / \mathrm{l} \mathrm{FSH}, 2.2 \mathrm{IU} / \mathrm{l} \mathrm{LH}$, $147 \mathrm{pmol} / \mathrm{l} \mathrm{E}_{2}, 4 \mathrm{pmol} / \mathrm{l}$ early morning fasting adrenocorticotropin (ACTH; normal: $\leq 10 \mathrm{pmol} / \mathrm{l}), 127 \mathrm{nmol} / \mathrm{l}$ random cortisol (normal: $120-535 \mathrm{nmol} / \mathrm{l}$ ), $11.4 \mathrm{nmol} / \mathrm{l}$ insulin-like growth factor 1 (normal: $13.3-42.6 \mathrm{nmol} / \mathrm{l}$ ), and $2.5 \mu \mathrm{g} / \mathrm{l}$ prolactin (normal: $3.3-26.7 \mu \mathrm{g} / \mathrm{l}$ ). Serum sodium, potassium, and chloride levels were normal. A clomiphene challenge test was administered, wherein $100 \mathrm{mg}$ of clomiphene was given for 5 days. Five days after completing the clomiphene, serum FSH and $\mathrm{E}_{2}$ levels were $3.7 \mathrm{IU} / \mathrm{l}$ and $<73.4 \mathrm{pmol} / \mathrm{l}$ respectively, which confirmed a pituitary gonadotropin production dysfunction. An insulininduced hypoglycemic stress test of the adrenal gland resulted in the serum levels that are shown in Table 1. Cortisol levels did not increase with induced hypoglycemia, as would normally occur. The patient did become symptomatic; however, adequate hypoglycemia was not obtained. A magnetic resonance imaging (MRI) exam showed a small, partially degenerated pituitary gland consistent with an empty sella as well as a thin hypothalamic stalk.

\section{Treatment}

Although doing so was debatable, oral glucocorticoid therapy was initiated in the form of $12.5 \mathrm{mg}$ Cortizone to be taken orally twice daily, because all other attempts to 
Table 1 Insulin bolus-induced hypoglycaemic changes in serum cortisol levels.

\begin{tabular}{|c|c|c|c|c|c|c|c|}
\hline & \multicolumn{7}{|c|}{ Time (min) } \\
\hline & -15 & 15 & 30 & 45 & 60 & 90 & 120 \\
\hline Serum cortisol levels (nmol/l) & 221 & 189 & 173 & 162 & 182 & 178 & 180 \\
\hline Serum insulin levels (pmol/l) & 120 & & 965 & & 992 & 1057 & 335 \\
\hline Serum glucose levels (mmol/l) & 12.5 & 14.2 & 10.4 & 9.2 & 7.4 & 5.9 & 4.8 \\
\hline
\end{tabular}

alter in any way the patient's endogenous cortisol levels failed. Diabetic control was augmented.

\section{Outcome and follow-up}

In order to explain the present patient's pituitary dysfunction, we hypothesized that she had a cerebrovascular accident at the level of the circle of Willis that had resulted from the vaso-occlusive effects of severe preeclampsia and the intake of a postpartum dopamine agonist.

\section{Discussion}

Bromocriptine acts as a dopamine agonist, and its side effects, which are mostly minor, include orthostatic hypotension, nausea, and headaches. Although it is rare, vasospasm has been described as being related to the use of bromocriptine, particularly in the postpartum period (2). Katz et al. (5) reported two cases of cerebrovascular accidents that presented several days after the use of bromocriptine. They concluded that the exact mechanism of the events that led to the cerebral accidents remained unclear, but they questioned the safety of bromocriptine use for lactation suppression. Kim et al. (4) reported a similar case of postpartum reversible cerebral vasoconstriction syndrome in a patient following bromocriptine use in the peripartum period. The postpartum period has been associated with an elevated risk for thromboembolic events (1). Postpartum cerebral vascular thrombosis has also been associated with severe preeclampsia (2). One case documented pituitary apoplexy (intra-pituitary hemorrhage) as a result of severe preeclampsia and HELLP syndrome with a resultant low platelet count and a risk for spontaneous bleeding (6). But to date there have been no cases described in the literature of hypopituitarism that resulted from a non-hemorrhagic, vaso-occlusive event, regardless of whether they occurred in the peripartum period or in a non-pregnancy state. In none of the cases in the literature has there been a report of pituitary damage related to preeclampsia without hemorrhage.

The present patient not only presented with secondary amenorrhea in the absence of other puerperal risk factors, but she also had radiologic imaging suggestive of injury at the pituitary level (7). The pituitary undergoes many anatomic and physiologic changes during pregnancy, mainly to accommodate enlarging lactotrophs, which makes it vulnerable to many pregnancy-related disorders (7). Defects at the level of the hypophysis are best visualized on MRI. A partially empty sella can signify a primary defect in the pituitary that is related to a defect in cerebrospinal fluid pressure or a secondary defect that is related to the removal or treatment of a mass, mass infarct, or pituitary apoplexy. A partially empty sella can also be seen in Sheehan's syndrome or lymphocytic hypophysitis (8) (9). According to the MRI report in the present study, the pituitary was found to have a thin, not thickened, pituitary stalk, which makes the differential of lymphocytic hypophysitis less likely. Moreover, the lack of a history of postpartum hemorrhage invalidates Sheehan's syndrome. The occurrence of amenorrhea was temporally associated with severe preeclampsia combined with the use of a postpartum dopamine agonist. The CT scan at the time of the incident confirmed a normal pituitary gland, which ruled out hemorrhage into a pituitary macroadenoma apoplexy. Vasospasm was diffusely present, which supported the conclusion of peripartum vasospasm at the pituitary level to explain our patient's symptomatology. However, the fact that the patient did not have an MRI exam in the peripartum period is a limitation. There has been a suggestion in the literature that the sensitivity of MRI, particularly in non-hemorrhage pituitary apoplexy, appears to be superior to that of CT scans (10) (11).

Hypopituitarism can have a variety of presentations. Symptoms vary according to the degree of hormonal deficiency and can be partial or total and acute or chronic. As a general rule, gonadotropins and growth hormones (GH) are more likely to be affected than ACTH and TSH are. Prolactin deficiency, which was present in our patient, is usually a rare manifestation and appears after global pituitary dysfunction (8). GH production was also suppressed in the present case. In spite of the symptomatology, the ACTH stimulation in this patient did not increase in response to insulin-induced hypoglycemia. However, the level of the hypoglycemia was insufficient 
to confirm a diagnosis of ACTH production failure. It is fascinating that the patient's serum cortisol levels remained unchanged in spite of the symptomatology, though. The symptomatology was likely caused by poorly controlled diabetes mellitus type 2 .

A combination of low $\mathrm{FSH}, \mathrm{LH}$, and $\mathrm{E}_{2}$ will prompt a diagnosis of hypogonadotropic hypogonadism (HH). The clomiphene challenge test is a commonly used test to confirm HH. When this test was administered to the present patient, she failed to produce gonadotropins, which once again confirmed a lesion at the pituitary level.

Infarction of a pituitary macroadenoma with a resultant pituitary hemorrhage can result in pituitary injury (12). No macro- or microadenomas of the pituitary gland were present in our patient. Traditionally, empty sella syndrome resulting from macroadenoma infarction and the resultant herniation of the meninges into the sella turcica and compression of the pituitary results in maintained pituitary function or a minimal loss of hormone production (12). Although panhypopituitarism can occur with empty sella syndrome, the macroadenoma that would have been required to initiate the event was not present (12). Lastly, up to $80 \%$ of patients with empty sella syndrome report chronic headaches, which were lacking in the present case (12). Although diabetes mellitus is associated with increased vascular disease, it has not been described as a cause of severe pituitary hypofunction because of the protection provided by the many vessels that feed the circle of Willis. Rarely, infiltrative diseases, such as sarcoidosis or lymphoma, or radiation therapy have been shown to damage the pituitary gland, although evidence of these diseases was lacking in the present case.

In the medical literature, pituitary injury resulting from a hemorrhagic peripartum event is called Shehan's syndrome, whereas a hemorrhagic non-pregnancy event (usually a car accident or other severe trauma) is called Simon's syndrome. To maintain continuity, we propose that pituitary injury resulting from a non-hemorrhagic vaso-occlusive event be called Dahan's syndrome.

It cannot be known if the damage caused to the pituitary resulted from preeclampsia, the use of a dopamine agonist postpartum, or a combination of both. The literature lists the use of dopamine agonists as being more common in patients who present with pituitary hypofunction (apoplexy) and a history of pituitary masses (13). However, the mechanism of pituitary injury was thought to be the result of tumor infarct, and the present case suggests that it may be a result of vascular spasm (13). Spasms are more likely to cause injury at the pituitary gland level in people with small microadenomas of 1-3 $\mathrm{mm}$ in size when they are on dopamine agonists than infarction is. Pituitary injury in these situations has been previously noted (13). It should be recognized that that the six vessels supply the circle of Willis from their most distal points. It could be hypothesized that uniform vasospasm of all of these vessels caused by a pathologic condition or medication could spare the brain but provide inadequate blood flow to the pituitary gland.

In short, many endocrine disorders can lead to amenorrhea. The differential diagnosis of secondary amenorrhea can be vast. In the present case, the investigation led to a hypothesis of postpartum pituitary hypofunction and damage secondary to vasospasm that was caused by a combination of severe preeclampsia or the use of a postpartum dopamine agonist. This makes the present case the first of its kind in the literature, and it has resulted in the coining of the term Dahan's syndrome.

Declaration of interest

The authors declare that there is no conflict of interest that could be perceived as prejudicing the impartiality of the research reported.

\section{Funding}

This research did not receive any specific grant from any funding agency in the public, commercial or not-for-profit sector.

\section{Patient consent}

Written informed consent was obtained from the patient for the publication of this case report.

\section{Author contribution statement}

All three authors were involved in the investigation and work up of this patient. All three authors participated in the writing of this case report. Dr M H Dahan was the primary physician, Dr D V Morris was the referral endocrinologist from Dr M H Dahan, and Dr A Kuriya was the resident on the case.

\section{References}

1 Kamel H, Navi BB, Sriram N, Hovsepian DA, Devereux RB \& Elkind MSV 2014 Risk of thrombotic event after the 6-week postpartum period. New England Journal of Medicine 370 1307-1315. (doi:10.1056/ NEJMoa1311485

2 Witlin AG, Mattar F \& Sibai B 2000 Postpartum stroke: a twenty-year experience. American Journal of Obstetrics and Gynecology 183 83-88. (doi:10.1016/S0002-9378(00)70397-8)

3 Hovsepian DA, Sriram N, Kamel H, Fink ME \& Navi BB 2014 Acute cerebrovascular disease occurring after hospital discharge for labor and delivery. Stroke 45 1947-1950. (doi:10.1161/STROKEAHA.114. 005129) 
4 Kim YS, Baek W, Kim J, Kim HY \& Lee YJ 2012 Delayed ischemic stroke associated with bromocriptine-induced reversible cerebral vasoconstriction syndrome. Neurological Sciences 34 409-411. (doi:10.1007/ s10072-012-1015-z)

5 Katz M, Kroll D, Pak I, Osimoni A \& Hirsch M 1985 Puerperal hypertension, stroke, and seizures after suppression of lactation with bromocriptine. Obstetrics and Gynecology 66 822-824.

6 Murao K, Imachi H, Muraoka T \& Ishida T 2011 Hemolysis, elevated liver enzymes, and low platelet count (HELLP) syndrome with pituitary apoplexy. Fertility and Sterility 96 260-261. (doi:10.1016/j.fertnstert.2011.05.012)

7 Sheriff FG, Howlett WP \& Kilonzo KG 2012 Post-partum pituitary insufficiency and livedo reticularis presenting a diagnostic challenge in a resource limited setting in Tanzania: a case report, clinical discussion and brief review of existing literature. BMC Endocrine Disorders 124 . (doi:10.1186/1472-6823-12-4)

8 Karaca Z, Tanriverdi F, Unluhizarci K \& Kelestimur F 2009 Empty sella may be the final outcome in lymphocytic hypophysitis. Endocrine Research 34 10-17. (doi:10.1080/07435800902841306)
9 Glezer A \& Bronstein MD 2012 Pituitary autoimmune disease: nuances in clinical presentation. Endocrine 42 72-74. (doi:10.1007/s12020-0129654-7)

10 Boellis A, di Napoli A, Romano A \& Bozzao A 2014 Pituitary apoplexy: an update on clinical and imaging features. Insights into Imaging $\mathbf{5}$ 753-762. (doi:10.1007/s13244-014-0362-0)

11 Randeva HS, Schoebel J, Byrne J, Esiri M, Adams CB \& Wass JA 1999 Classical pituitary apoplexy: clinical features, management and outcome. Clinical Endocrinology 51 181-188. (doi:10.1046/j.1365-2265. 1999.00754.x)

12 Guitelman M, Garcia Basavilbaso N, Vitale M, Chervin A, Katz D, Miragaya K, Herrera J, Cornalo D, Servidio M, Boero L et al. 2013 Primary empty sella (PES): a review of 175 cases. Pituitary 2 270-274. (doi:10.1007/s11102-012-0416-6)

13 Vargas G, Gonzalez B, Guinto G, Mendoza V, López-Félix B, Zepeda E \& Mercado M 2014 Pituitary apoplexy in nonfunctioning pituitary macroadenomas: a case-control study. Endocrine Practice 20 1274-1280. (doi:10.4158/EP14120.OR)

Received in final form 19 April 2015

Accepted 23 April 2015 